\title{
Intersection theory and separation exponent in complex analytic geometry
}

\author{
by Ewa Cygan (Kraków)
}

\begin{abstract}
We consider the intersection multiplicity of analytic sets in the general situation. We prove that it is a regular separation exponent for complex analytic sets and so it estimates the Łojasiewicz exponent. We also give some geometric properties of proper projections of analytic sets.
\end{abstract}

1. Introduction. The aim of this paper is to find a connection between two indices which characterize locally the intersection of analytic sets: intersection multiplicity and separation exponent. In $[\mathrm{CT}],\left[\mathrm{T}_{3}\right]$ such a relation has been established in two particular cases: proper intersection and isolated intersection of analytic sets.

The definition of the intersection multiplicity in the improper case, proposed recently by $\mathrm{P}$. Tworzewski $\left[\mathrm{T}_{2}\right]$, raises the natural question about a generalization of these results. The main theorem of this paper (Thm. 4.4) confirms the hypothesis that the intersection multiplicity is a separation exponent for analytic sets. Moreover, some geometric properties of proper projections of analytic sets are given (Section 3), which can represent interesting tools in the investigation of geometric characterizations of analytic sets.

The main result presented here has already found nice applications in estimating the Łojasiewicz exponent at infinity for polynomial mappings; in particular, using it one can improve Kollár's well-known results (see [CKT], $[\mathrm{K}])$.

2. Intersection multiplicity. For the convenience of the reader we compile in this section some basic notions of intersection theory (see [ATW], $[\mathrm{Ch}],[\mathrm{D}],\left[\mathrm{T}_{2}\right]$ for more details).

1991 Mathematics Subject Classification: 32B10, $14 \mathrm{C} 17$.

Key words and phrases: multiplicity of proper and improper intersection, exponent of regular separation.

Supported by KBN Grant 2 P03A 06108. 
I. Analytic cycles and their multiplicities. In this paper analytic means complex analytic, and manifold means a complex manifold satisfying the second axiom of countability. Let $M$ be a manifold of dimension $m$. An analytic cycle on $M$ is a formal sum

$$
A=\sum_{j \in J} \alpha_{j} Z_{j}
$$

where $\alpha_{j} \neq 0$ for $j \in J$ are integers and $\left\{Z_{j}\right\}_{j \in J}$ is a locally finite family of pairwise distinct irreducible analytic subsets of $M$.

The analytic set $\bigcup_{j \in J} Z_{j}$ is called the support of the cycle $A$ and is denoted by $|A|$. If all the components of $A$ have the same dimension $k$, then $A$ is called a $k$-cycle. We say that $A$ is positive if $\alpha_{j}>0$ for all $j \in J$.

We consider the natural extension of the local multiplicity of analytic sets. Namely, if $a \in M$ and $\nu\left(Z_{j}, a\right)$ denotes the multiplicity of $Z_{j}$ at the point $a$ (see [D], p. 194), then the sum

$$
\nu(A, a)=\sum_{j \in J} \alpha_{j} \nu\left(Z_{j}, a\right)
$$

is well defined and called the multiplicity of the cycle $A$ at the point $a$.

There exists a unique decomposition

$$
A=T_{(m)}+T_{(m-1)}+\ldots+T_{(0)},
$$

where $T_{(j)}$ is a $j$-cycle for $j=0, \ldots, m$. For our purpose it will be useful to introduce the notion of extended multiplicity of $A$ at $a$ by the formula

$$
\widetilde{\nu}(A, a)=\left(\nu\left(T_{(m)}, a\right), \ldots, \nu\left(T_{(0)}, a\right)\right) \in \mathbb{Z}^{m+1} .
$$

Denote by $\nu(A)$ and $\widetilde{\nu}(A)$ the functions

$$
\nu(A): M \ni x \rightarrow \nu(A, x) \in \mathbb{Z}, \quad \widetilde{\nu}(A): M \ni x \rightarrow \widetilde{\nu}(A, x) \in \mathbb{Z}^{m+1} .
$$

Observe that $\nu(A, x)=\widetilde{\nu}(\widehat{A, x})$, where $\widehat{\nu}$ denotes the sum of the coordinates of $\nu \in \mathbb{Z}^{m+1}$.

II. Proper intersections and regular directions. Let now $X$ and $Y$ be pure dimensional analytic subsets of $M$. We say that $X$ and $Y$ meet properly on $M$ if $\operatorname{dim}(X \cap Y)=\operatorname{dim} X+\operatorname{dim} Y-m$. Then we have the intersection product $X \cdot Y$ which is an analytic cycle on $M$ defined by the formula

$$
X \cdot Y=\sum_{Z} i(X \cdot Y, Z) Z,
$$

where the summation extends over all the analytic components $Z$ of $X \cap Y$ and $i(X \cdot Y, Z)$ denotes the intersection multiplicity along $Z$ in the sense of Draper ([D], Def. 4.5; cf. [W]). Such multiplicities are positive integers.

Consider now the special situation when $M$ is a neighbourhood of zero in a normed complex vector space $N$. Take a pure $k$-dimensional analytic 
subset $Z$ in $M$ and a linear subspace $\Lambda$ of dimension $m-k$ such that zero is an isolated point of $Z \cap \Lambda$. We say that $\Lambda$ is a regular direction for $Z$ in $N$ if $i(Z \cdot \Lambda, 0)=\nu(Z, 0)$. Recall that $\Lambda$ is a regular direction for $Z$ if and only if $\Lambda \cap C(Z, 0)=\{0\}$, where $C(Z, 0)$ is the tangent cone to $Z$ at zero ([D], Thm. 6.3). Hence the subset of all regular directions for $Z$ in $N$ is open and dense in the grassmannian manifold $G^{m-k}(N)$.

III. Intersections of analytic sets with submanifolds. Let $M$ be an $m$ dimensional manifold. Fix a closed $s$-dimensional submanifold $S$ of $M$ and an open subset $U$ of $M$ such that $U \cap S \neq \emptyset$. For a given cycle $A=$ $\sum_{j \in J} \alpha_{j} Z_{j}$ analytic on $M$, by its part supported by $S$ we mean the cycle $A^{S}=\sum_{j \in J, Z_{j} \subset S} \alpha_{j} Z_{j}$. Denote by $\mathcal{H}(U)$ the set of all $\mathcal{H}:=\left(H_{1}, \ldots, H_{m-s}\right)$ satisfying the following conditions:

(1) $H_{j}$ is a smooth hypersurface of $U$ containing $U \cap S$ for $j=1, \ldots, m-s$,

(2) $\bigcap_{j=1}^{m-s} T_{x}\left(H_{j}\right)=T_{x} S$ for each $x \in U \cap S$.

For a given analytic subset $Z$ of $M$ of pure dimension $k$ we denote by $\mathcal{H}(U, Z)$ the set of all $\mathcal{H} \in \mathcal{H}(U)$ such that $((U \backslash S) \cap Z) \cap H_{1} \cap \ldots \cap H_{j}$ is an analytic subset of $U \backslash S$ of pure dimension $k-j$ (or empty) for $j=1, \ldots, k$.

Following $\left[\mathrm{T}_{2}\right]$ we present here an algorithm which produces for every $\mathcal{H} \in \mathcal{H}(U, Z)$ an analytic cycle $Z \cdot \mathcal{H}$ in $S \cap U$. At each step of the algorithm we get a cycle $Z_{i}=Z_{i}^{S}+\left(Z_{i}-Z_{i}^{S}\right)$. Denote by $i_{\mathcal{H}} \in\{0, \ldots, m-s\}$ the first index for which $\left|Z_{i_{\mathcal{H}}}-Z_{i_{\mathcal{H}}}^{S}\right|=\emptyset$.

(2.1) Algorithm.

SteP 0 . Let $Z_{0}=Z \cap U$. Then $Z_{0}=Z_{0}^{S}+\left(Z_{0}-Z_{0}^{S}\right)$, where $Z_{0}^{S}$ is the part of $Z_{0}$ supported by $S \cap U$.

SteP 1. Let $Z_{1}=\left(Z_{0}-Z_{0}^{S}\right) \cdot H_{1}$. Then $Z_{1}=Z_{1}^{S}+\left(Z_{1}-Z_{1}^{S}\right)$, where $Z_{1}^{S}$ is the part of $Z_{1}$ supported by $S \cap U$.

SteP 2. Let $Z_{2}=\left(Z_{1}-Z_{1}^{S}\right) \cdot H_{2}$. Then $Z_{2}=Z_{2}^{S}+\left(Z_{2}-Z_{2}^{S}\right)$, where $Z_{2}^{S}$ is the part of $Z_{2}$ supported by $S \cap U$.

STEP $i_{\mathcal{H}}$. Let $Z_{i_{\mathcal{H}}}=\left(Z_{i_{\mathcal{H}}-1}-Z_{i_{\mathcal{H}}-1}^{S}\right) \cdot H_{i_{\mathcal{H}}}$. Now we have the decomposition $Z_{i_{\mathcal{H}}}=Z_{i_{\mathcal{H}}}^{S}+\left(Z_{i_{\mathcal{H}}}-Z_{i_{\mathcal{H}}}^{S}\right)$, and $\left|Z_{i_{\mathcal{H}}}-Z_{i_{\mathcal{H}}}^{S}\right| \cap S=\emptyset$.

We call the positive analytic cycle $Z \cdot \mathcal{H}=Z_{0}^{S}+Z_{1}^{S}+\ldots+Z_{i_{\mathcal{H}}}^{S}$ in $S \cap U$ the result of the above algorithm.

At an arbitrary point $a \in S$ the set $Z$ can be characterized by two indices $g(a)$ and $p(a)$ which we now define. Let

$$
\widetilde{g}(a)=\widetilde{g}(Z, S)(a):=\min _{\operatorname{lex}}\{\widetilde{\nu}(Z \cdot \mathcal{H}, a): \mathcal{H} \in \mathcal{H}(U, Z), a \in U\} \in \mathbb{N}^{s+1}
$$

and $g(a)=g(Z, S)(a)=(\widehat{\widetilde{g}(a)})$. The number $g(a)$ is called the index of intersection of $Z$ and $S$ at $a$ (see [T $\mathrm{T}_{2}$ ], Def. 4.2). 
From our point of view the following index is much more interesting for applications in regular separation.

Definition 2.2. For $a \in S$ we call

$$
p(a)=p(Z, S)(a):=\min \{\nu(Z \cdot \mathcal{H}, a): \mathcal{H} \in \mathcal{H}(U, Z), a \in U\} \in \mathbb{N}
$$

the index of contact of $Z$ and $S$ at $a$.

Observe that we always have $p(a) \leq g(a)$.

III. Intersection of analytic sets-general case. Let $X$ and $Y$ be irreducible analytic subsets of an $m$-dimensional manifold $M$ and let $a \in M$. By standard diagonal construction the multiplicity of intersection of $X$ and $Y$ at $a$ is defined to be

$$
d(a)=d(X, Y)(a)=g\left(X \times Y, \Delta_{M},(a, a)\right) .
$$

The intersection product of $X$ and $Y$ is a unique analytic cycle $X \bullet Y$ in $M$ such that $\nu(X \bullet Y)=d(X, Y)$ (see [ $\left.\mathrm{T}_{2}\right]$, Def. 6.3).

The above definition can be naturally extended to the case of the intersection of a finite number of irreducible analytic subsets and next to the case of arbitrary analytic cycles by multilinearity.

3. Special properties of proper projections of analytic sets. Let $M$ be a complex inner product space of dimension $m$ and $\Omega$ a neighbourhood of zero in $M$. Consider a positive $k$-cycle $A$ in $\Omega$. If $\Lambda$ is an $(m-k)$ dimensional linear subspace of $M$ such that $\Lambda \cap|A|=\{0\}$ then there exists a connected neighbourhood of zero in $M=\Lambda^{\perp}+\Lambda=\Lambda^{\perp} \times \Lambda$ of the form $G=U \times W \subset \Omega$, where $U$ and $W$ are balls in the spaces $\Lambda^{\perp}, \Lambda$ respectively, such that the natural projection $\left.\pi_{\Lambda}\right|_{|A| \cap G}:|A| \cap G \rightarrow U$ is a $p$-sheeted branched covering with $p=\nu(|A| \cdot \Lambda, 0)$, i.e.

(1) $\left.\pi_{\Lambda}\right|_{|A|}$ is surjective and proper,

(2) for every $x \in U$ the fibre $\left(\left.\pi_{\Lambda}\right|_{|A|}\right)^{-1}(x)$ is finite,

(3) there exists a proper analytic subset $S$ of $U$ such that $\left.\pi_{\Lambda}\right|_{|A|}$ is locally biholomorphic on $|A| \backslash \pi_{\Lambda}^{-1}(S)$ and

$$
\begin{array}{ll}
\#\left(\left.\pi_{\Lambda}\right|_{|A|}\right)^{-1}(x)=p & \text { if } x \in U \backslash S, \\
\#\left(\left.\pi_{\Lambda}\right|_{|A|}\right)^{-1}(x)<p & \text { if } x \in S .
\end{array}
$$

The set $S$ is called the critical set of the branched covering $\left.\pi_{\Lambda}\right|_{|A|}$, and $U \backslash S$ its regular set.

Without loss of generality we can assume that all the components of $A$ pass through zero and $G=\Omega$. For each component $Z$ of $A$ the projection $\left.\pi_{\Lambda}\right|_{Z}$ is also a branched covering and we denote its multiplicity by $p_{\Lambda, Z}$. 
For every cycle $A$ we now define a certain useful real function on $G$. First for each component $Z$ of $A$ we define

$$
d_{G, \Lambda, Z}(z)=\prod_{i=1}^{p_{\Lambda, Z}}\left|z-z_{i}\right| \quad \text { for }\left(\pi_{\Lambda} \mid Z\right)^{-1}\left(\pi_{\Lambda}(z)\right)=\left\{z_{1}, \ldots, z_{p_{\Lambda, Z}}\right\}
$$

where $z_{i}$ are counted with their multiplicities. Next for the cycle $A=$ $\sum_{Z} \alpha_{Z} Z$ we put

$$
d_{G, \Lambda, A}(z)=\prod_{Z} d_{G, \Lambda, Z}^{\alpha_{z}}(z)
$$

Further we consider the germ of $d_{G, \Lambda, A}$ at zero, denoted by $\mathbf{d}_{\Lambda, A}$. For representatives of $\mathbf{d}_{\Lambda, A}$ we use the notation $d_{\Lambda, A}$.

The next remark will be used in the proof of the main theorem.

REMARK 3.1. If $M^{\prime}$ is a linear subspace of $M, \Lambda \subset M^{\prime}$ and the intersection of $A$ and $M^{\prime}$ is proper then for $A^{\prime}=A \cdot M^{\prime}$ considered as a positive cycle on $G^{\prime}=G \cap M^{\prime}$ we have $d_{G^{\prime}, \Lambda, A^{\prime}}(z)=d_{G, \Lambda, A}(z)$ for $z \in G^{\prime}$.

Proof. Notice that for all $z \in G^{\prime}$ we have $d_{G, \Lambda, A}(z)=\prod_{i=1}^{r}\left|z-z_{i}\right|^{\alpha_{i}}$ if $A \cdot(\Lambda+z)=\sum_{i=1}^{r} \alpha_{i}\left\{z_{i}\right\}$. As $A \cdot M(\Lambda+z)=\left(A \cdot \cdot_{M} M^{\prime}\right) \cdot M^{\prime}(\Lambda+z)\left(\left[\mathrm{TW}_{2}\right]\right.$, Thm. 2.2), the equality follows.

Consider now a non-zero linear form $l: \Lambda \rightarrow \mathbb{C}$ and define the linear mapping

$$
L: M=\Lambda^{\perp} \times \Lambda \ni(x, y) \rightarrow(x, l(y)) \in \Lambda \times \mathbb{C} .
$$

Note that $\operatorname{ker} L \cap|A|=\{0\}$ and for each component $Z$ of $A$ we get some standard properties:

(i) $\left.L\right|_{Z}$ is proper $\left(\right.$ see $\left.\left[\mathrm{TW}_{1}\right]\right)$,

(ii) $Z_{L}=L(Z)$ is an irreducible analytic subset of $U \times l(W)$ of pure dimension $k$,

(iii) $\left.L\right|_{Z}: Z \rightarrow Z_{L}$ is a $\mu_{L, Z}$-sheeted analytic covering (see [D]),

(iv) the natural projection $\left.\pi_{\Lambda}\right|_{Z_{L}}: Z_{L} \ni(x, t) \rightarrow x \in U$ is a $p_{Z_{L}}$-sheeted analytic covering for some $p_{Z_{L}} \in \mathbb{N}$.

In consequence there exists a unique system of functions $\alpha_{1, Z}, \ldots, \alpha_{p_{Z_{L}}, Z}$ holomorphic on $U$ such that

$$
Z_{L}=\left\{(x, t) \in U \times l(W): P_{l, Z}(x, t)=0\right\},
$$

where $P_{l, Z}(x, t)=t^{p_{Z_{L}}}+\alpha_{1, Z}(x) t^{p_{Z_{L}}-1}+\ldots+\alpha_{p_{Z_{L}}, Z}(x)$.

For a cycle $A$ on $G$ we now introduce a holomorphic function determined by the linear form $l$ as follows:

$$
F_{G, l, A}(z)=\prod_{Z}\left(P_{l, Z}(L(z))\right)^{\mu_{L, Z} \cdot \alpha_{Z}} .
$$

We denote by $\mathbf{F}_{l, A}$ the germ of $F_{G, l, A}$ at zero, and by $F_{l, A}$ its representatives. 
It is useful to introduce another holomorphic mapping. Let $l_{1}, \ldots, l_{r}$ be non-zero linear forms on $\Lambda$. We put

$$
F_{G,\left(l_{1}, \ldots, l_{r}\right), A}(z)=\left(F_{G, l_{1}, A}(z), \ldots, F_{G, l_{r}, A}(z)\right) \text { for } z \in G .
$$

The germ of this mapping at zero will be denoted by $\mathbf{F}_{\left(l_{1}, \ldots, l_{r}\right), A}$, and its representatives by $F_{\left(l_{1}, \ldots, l_{r}\right), A}$.

To simplify the notation, an inequality for germs of real functions will mean the inequality between some of their representatives.

Let us now recall the following general lemma (see $[\mathrm{CT}]$ ).

Lemma 3.2. Suppose that $n, d$ are positive integers, $r=(n-1) d+1$ and $l_{1}, \ldots, l_{r}$ are linear forms on $\Lambda$ such that $l_{i_{1}}, \ldots, l_{i_{n}}$ are linearly independent for $i_{1}, \ldots, i_{n} \in\{1, \ldots, r\}$ such that $i_{s} \neq i_{t}$ for $s \neq t$. Define

$$
\Phi: \Lambda^{d} \ni\left(v_{1}, \ldots, v_{d}\right) \rightarrow\left(\prod_{i=1}^{d} l_{1}\left(v_{i}\right), \ldots, \prod_{i=1}^{d} l_{r}\left(v_{i}\right)\right) \in \mathbb{C}^{r} .
$$

Then there exists a positive constant $c>0$ such that $\left|\Phi\left(v_{1}, \ldots, v_{d}\right)\right| \geq$ $c\left|v_{1}\right| \ldots\left|v_{d}\right|$ for $v_{1}, \ldots, v_{d} \in \Lambda$.

The next lemma establishes relations between all the functions introduced before.

LEMMA 3.3. Let $l_{1}, \ldots, l_{r}$ be linear forms as in Lemma 3.2 with $n=$ $m-k$ and $d=\nu(\Lambda \cdot A, 0)$, and $l: \Lambda \rightarrow \mathbb{C}$ a non-zero linear form on $\Lambda$. Then there exist constants $c^{\prime}, c^{\prime \prime}>0$ such that

$$
c^{\prime}\left|\mathbf{F}_{l, A}\right| \leq \mathbf{d}_{\Lambda, A} \leq c^{\prime \prime}\left|\mathbf{F}_{\left(l_{1}, \ldots l_{r}\right), A}\right| .
$$

Proof. It suffices to show that for some constants $c^{\prime}, c^{\prime \prime}>0$,

$$
c^{\prime}\left|F_{G, l, A}(z)\right| \leq d_{G, \Lambda, A}(z) \leq c^{\prime \prime}\left|F_{G,\left(l_{1}, \ldots l_{r}\right), A}(z)\right| \quad \text { for } z \in G .
$$

Denote by $S$ the critical set of the analytic covering $\left.\pi_{\Lambda}\right|_{|A|}:|A| \rightarrow U$ and fix $z=(x, y) \in G$ with $x \in U \backslash S$. Let

$$
\left(\left.\pi_{\Lambda}\right|_{Z}\right)^{-1}(x)=\left\{\left(x, y_{1, Z}\right), \ldots,\left(x, y_{p_{\Lambda, Z}, Z}\right)\right\}
$$

for every component $Z$ of $|A|$. Applying Lemma 3.2 to the system $v_{1}, \ldots, v_{p}$ $\in \Lambda$ where each of the points $y-y_{1, Z}, \ldots, y-y_{p_{\Lambda, Z}, Z}$ is repeated $\alpha_{Z}$ times we get constants $c^{\prime}, c^{\prime \prime}>0$ such that

$$
c^{\prime}\left|\prod_{i=1}^{p} l\left(v_{i}\right)\right| \leq \prod_{i=1}^{p}\left|v_{i}\right| \leq c^{\prime \prime}\left|\left(\prod_{i=1}^{p} l_{1}\left(v_{i}\right), \ldots, \prod_{i=1}^{p} l_{r}\left(v_{i}\right)\right)\right| .
$$

So according to our definitions it follows that

$$
\begin{aligned}
& c^{\prime}\left|\prod_{Z}\left(\prod_{i=1}^{p_{\Lambda}, Z} l\left(y-y_{i, Z}\right)\right)^{\alpha_{Z}}\right| \\
& \leq d_{G, \Lambda, A}(z) \leq c^{\prime \prime}\left|\left(\prod_{Z}\left(\prod_{i=1}^{p_{\Lambda, Z}} l_{1}\left(y-y_{i, Z}\right)\right)^{\alpha_{Z}}, \ldots, \prod_{Z}\left(\prod_{i=1}^{p_{\Lambda, Z}} l_{r}\left(y-y_{i, Z}\right)\right)^{\alpha_{Z}}\right)\right|
\end{aligned}
$$


and after standard calculations we finally get

$$
c^{\prime}\left|F_{G, l, A}(z)\right| \leq d_{G, \Lambda, A}(z) \leq c^{\prime \prime}\left|F_{G,\left(l_{1}, \ldots l_{r}\right), A}(z)\right| \quad \text { for } z \in G \backslash(S \times W) .
$$

By continuity of all the functions considered we have the required inequalities on the whole $G$.

Lemma 3.4. Let $A, B$ be positive analytic $k$-cycles on $\Omega$ and $\Lambda$ an $(m-k)$-dimensional linear subspace of $M$ such that $\Lambda \cap|A|=\{0\}$ and $\operatorname{dim} C(|B|, 0) \cap \Lambda=l>0$. Then there exist $\Lambda_{1}, \ldots, \Lambda_{r} \in G^{m-k}(M)$ and $c>0$ such that

(1) $\operatorname{dim} C(|B|, 0) \cap \Lambda_{i}<l$,

(2) $\mathbf{d}_{\Lambda, A} \leq c \sum_{i=1}^{r} \mathbf{d}_{\Lambda_{i}, A}$.

Proof. Without loss of generality we can assume that $M=\mathbb{C}^{m}, \Lambda=$ $\{0\} \times \mathbb{C}^{m-k}=\left\{z \in \mathbb{C}^{m}: z_{1}=\ldots=z_{k}=0\right\}$ and $\operatorname{dim} C(|B|, 0) \cap \widetilde{\Lambda}=$ $\operatorname{dim} C(|B|, 0) \cap \Lambda=l$, where $\tilde{\Lambda}=\left\{z \in \mathbb{C}^{m}: z_{1}=\ldots=z_{k-1}=0\right\}$. Put $Z=C(|B|, 0) \cap \widetilde{\Lambda}$ and consider $S=\left\{\zeta \in G^{m-1}\left(\mathbb{C}^{m}\right): \operatorname{dim} Z \cap \zeta \geq l\right\}$.

According to $\left[\mathrm{T}_{1}\right]$, Lemma 4.12 , we know that $G^{m-1}\left(\mathbb{C}^{m}\right) \backslash S$ is an open and dense subset in the manifold $G^{m-1}\left(\mathbb{C}^{m}\right)$.

So it is possible to choose a system of hyperplanes $\zeta_{j}=\left\{z \in \mathbb{C}^{m}: l_{j}(z)\right.$ $=0\}, j=1, \ldots, r$, where $r=(m-k-1) \nu(A \cdot \Lambda, 0)+1$, satisfying the following conditions:

(1) $\zeta_{j}=\left\{z \in \mathbb{C}^{m}: l_{j}(z)=0\right\} \notin S$,

(2) every system of linear forms $\left.l_{j_{1}}\right|_{\Lambda}, \ldots,\left.l_{j_{m-k}}\right|_{\Lambda}$ is linearly independent for $j_{1}, \ldots, j_{m-k} \in\{1, \ldots, r\}$ provided $j_{s} \neq j_{t}$ for $s \neq t$.

Applying Lemma 3.3 to the subspace $\Lambda$ and the system $\left.l_{1}\right|_{\Lambda}, \ldots,\left.l_{r}\right|_{\Lambda}$ we get

$$
\mathbf{d}_{\Lambda, A} \leq c \sum_{j=1}^{r}\left|\mathbf{F}_{\left.l_{j}\right|_{\Lambda}, A}\right| .
$$

Now consider the subspaces $\Lambda_{j}=\zeta_{j} \cap \widetilde{\Lambda}$. For each of the epimorphisms $L_{j}: \mathbb{C}^{m} \ni\left(z_{1}, \ldots, z_{m}\right) \rightarrow\left(z_{1}, \ldots, z_{k},\left.l_{j}\right|_{\Lambda}\left(z_{k+1}, \ldots, z_{m}\right)\right) \in \mathbb{C}^{k+1}$ we have ker $L_{j} \subset \Lambda_{j}$. Since $\operatorname{dim}\left(\operatorname{ker} L_{j}\right)=m-k-1$ it is possible to choose for every $j \in\{1, \ldots, r\}$ a linear form $\widetilde{l}_{j}$ on $\Lambda_{j}$ such that $\operatorname{ker} L_{j}=\operatorname{ker} \widetilde{L}_{j}$ for $\widetilde{L}_{j}: \mathbb{C}^{m}=\Lambda_{j}^{\perp} \times \Lambda_{j} \ni(x, y) \rightarrow\left(x, \widetilde{l}_{j}(y)\right) \in \mathbb{C}^{k+1}$. Consequently, there exist linear isomorphisms $I_{j}: \mathbb{C}^{k+1} \rightarrow \mathbb{C}^{k+1}$ for which $\widetilde{L}_{j}=I_{j} \circ L_{j}$. Hence it is easy to see that for every component $Z$ of the cycle $A$ the multiplicities $\mu_{L_{j}, Z}$ and $\mu_{\widetilde{L}_{j}, Z}$ coincide. As the germs of $P_{\left.l_{j}\right|_{A}, Z}$ and $P_{\widetilde{l}_{j}, A} \circ I$ at zero in $\mathbb{C}^{k+1}$ generate the ideal of the germ of $L_{j}(Z)$, we get

$$
\left|\mathbf{F}_{\left.l_{j}\right|_{A}, A}\right| \leq \widetilde{c}\left|\mathbf{F}_{\widetilde{l}_{j}, A}\right| \text { for some } \widetilde{c}>0 .
$$


Combining now $(*)$ and $(* *)$ and applying once more Lemma 3.3 to each of the forms $\widetilde{l}_{j}$ we finally get $\mathbf{d}_{\Lambda, A} \leq c \sum_{j=1}^{r} \mathbf{d}_{\Lambda_{j}, A}$.

As $\operatorname{dim} C(|B|, 0) \cap \widetilde{\Lambda} \cap \zeta_{j}<l$ we obtain $\operatorname{dim} C(|B|, 0) \cap \Lambda_{j}<\operatorname{dim} C(|B|, 0)$ $\cap \Lambda$ and this completes the proof.

TheOrem 3.5. Let $A, B$ be positive analytic $k$-cycles on $\Omega$ and $\Lambda$ an $(m-k)$-dimensional linear subspace of $M$ with $\Lambda \cap|A|=\{0\}$. Then there exist $\Lambda_{1}, \ldots, \Lambda_{s} \in G^{m-k}(M)$ and $c>0$ such that

(1) $\Lambda_{1}, \ldots, \Lambda_{s}$ are regular directions for the cycle $B$ in $M$,

(2) $\mathbf{d}_{\Lambda, A} \leq c \sum_{i=1}^{s} \mathbf{d}_{\Lambda_{i}, A}(z)$.

Proof. Thanks to the characterization of regular directions in terms of the dimension of the intersection of $\Lambda$ with the tangent cone to $B$ (see Section 2), the assertion follows by repeated application of Lemma 3.4.

REMARK 3.6. Observe that the assertion of Theorem 3.5 can be formulated in a more convenient way:

There exist regular directions $\Lambda_{1}, \ldots, \Lambda_{s}$ for $B$, representatives $d_{\Lambda, A}$, $d_{\Lambda_{1}, A}, \ldots, d_{\Lambda_{s}, A}, c>0$ and a neighbourhood $G$ of zero in $M$ such that for every $z \in G$ there exists $i_{0} \in\{1, \ldots, s\}$ such that $d_{\Lambda, A}(z) \leq c d_{\Lambda_{i_{0}}, A}(z)$.

We call the subspace $\Lambda_{i_{0}}$ chosen in the above way the maximal subspace for the point $z$ where the following elements are supposed to be given: the subspace $\Lambda$, system $\Lambda_{1}, \ldots, \Lambda_{s}$, neighbourhood $G$ and constant $c$.

The following proposition, closely related to [JKS], Lemma 8, establishes the relations between the function $d_{\Lambda, A}$ and the distance to the support of $A$.

Proposition 3.7. Let $A$ be a positive analytic $k$-cycle on $\Omega$, and $\Lambda$ an $(m-k)$-dimensional linear subspace of $M$ such that $\Lambda \cap|A|=\{0\}$. If $d_{\Lambda, A}$ is a representative of the germ $\mathbf{d}_{\Lambda, A}$ then there exist a constant $c>0$ and $a$ neighbourhood $G$ of zero in $M$ such that

$$
c \varrho(z,|A|) \geq d_{\Lambda, A}(z) \geq \varrho(z,|A|)^{p}
$$

for $p=\nu(A \cdot \Lambda, 0)$ and $z \in G$.

Proof. Without loss of generality we can assume that $\Omega=G$, where $G$ is the neighbourhood of zero chosen at the beginning of this section. Suppose also that $\bar{B}(0,2 R) \subset G$ for some $R>0$. Note that the zero set of the function $F=F_{G,\left(l_{1}, \ldots, l_{r}\right), A}$ is just $|A|$.

By the mean value theorem there exists $\widetilde{c}>0$ such that $\left|F\left(z^{\prime}\right)-F\left(z^{\prime \prime}\right)\right| \leq$ $\widetilde{c}\left|z^{\prime}-z^{\prime \prime}\right|$ if $z^{\prime}, z^{\prime \prime} \in B(0,2 R)$. For $z \in B(0, R)$ there is $w \in|A| \cap B(0,2 R)$ such that $\varrho(z,|A|)=|z-w|$. Then, by Lemma 3.3,

$$
\varrho(z,|A|)=|z-w| \geq \widetilde{c}^{-1}|F(z)-F(w)| \geq c^{\prime} d_{\Lambda, A}(z) .
$$

As the second inequality follows directly from the definition of $d_{\Lambda, A}$ this ends the proof. 
4. Separation exponent of analytic sets. We first repeat some basic facts on regular separation, thus making our exposition self-contained. For the proofs we refer the reader to $[\mathrm{CT}]$ ( $\mathrm{cf}$. $\left[\mathrm{E}_{1}\right]-\left[\mathrm{E}_{3}\right]$ ).

Let $M$ be an $m$-dimensional normed complex vector space and $X, Y$ closed sets in an open subset $G$ of $M$. For $p \in[1, \infty)$, we say that $X$ and $Y$ are $p$-separated at $a \in G$ if $a \in X \cap Y$ and

$$
\varrho(z, X)+\varrho(z, Y) \geq c \varrho(z, X \cap Y)^{p}
$$

in a neighbourhood of $a$, for some $c>0$.

LEMma 4.1. Let $H_{1} \subset G$ and $H_{2}$ be open subsets of normed, finitedimensional complex vector spaces and let $f: H_{1} \rightarrow H_{2}$ be a biholomorphism. Then closed subsets $X$ and $Y$ of $G$ are p-separated at a point $a \in H_{1}$ if and only if $f\left(X \cap H_{1}\right)$ and $f\left(Y \cap H_{1}\right)$ are p-separated at $f(a)$.

According to the above lemma we can consider $p$-separation for closed subsets of complex manifolds. Namely, we say that closed subsets $X$ and $Y$ of an $m$-dimensional complex manifold $M$ are $p$-separated at $a \in M$ if for some (and hence every) chart $\varphi: \Omega \rightarrow G \subset \mathbb{C}^{m}$ such that $a \in \Omega$, the sets $\varphi(X \cap \Omega)$ and $\varphi(Y \cap \Omega)$, closed in $G$, are $p$-separated at $\varphi(a)$.

Lemma 4.2. Let $G$ be an open subset of a normed finite-dimensional complex vector space. Then, for $p \geq 1, X$ and $Y$ are $p$-separated at $a$ if and only if there exists a neighbourhood $U$ of $a$ and $c>0$ such that

$$
\varrho(x, Y) \geq c \varrho(x, X \cap Y)^{p} \quad \text { for } x \in X \cap U .
$$

Lemma 4.3. Let $M$ be a complex manifold. If $a \in M$ and $p \geq 1$ then the following conditions are equivalent:

(1) $X$ and $Y$ are p-separated at a,

(2) $X \times Y$ and $\Delta_{M}$ are p-separated at $(a, a)$,

where $\Delta_{M}=\left\{(x, x) \in M^{2}: x \in M\right\}$ is the diagonal in $M^{2}$.

We can now formulate our main result.

THeOREM 4.4. Let $Z$ be a pure $k$-dimensional analytic subset of a complex manifold $M, S$ a closed $s$-dimensional submanifold of $M$ and $a \in Z \cap S$. Then $Z$ and $S$ are $p$-separated for $p=p(Z, S)(a)$.

P r o of. First choose a neighbourhood $U$ of $a$ and a system of hypersurfaces $\mathcal{H}=\left(H_{1}, \ldots, H_{m}\right) \in \mathcal{H}(U, Z)$ such that for every $i \in\{1, \ldots, m-s\}$ all the components of $\left|Z_{i}^{S}\right|$ pass through $a$. Define $n=i_{\mathcal{H}}$.

To prove the theorem we will show, using Algorithm (2.1), that the sets $Z$ and $S$ are $p_{\mathcal{H}}=\nu(Z \cdot \mathcal{H}, a)$-separated. Applying an appropriate chart we can assume that $Z, S$ are subsets of $\mathbb{C}^{m}, a=0$ and $H_{1}, \ldots, H_{n}$ can be regarded as linear subspaces. 
Fix a linear subspace $\Lambda_{n}$ in $H_{1} \cap \ldots \cap H_{n}$ which is a regular direction for $\left|Z_{n}^{S}\right|$ in $H_{1} \cap \ldots \cap H_{n}$.

We will choose a special system of linear subspaces in $\mathbb{C}^{m}$ in $n$ steps.

(1) Applying Theorem 3.5 for the cycles $A=Z_{n-1}-Z_{n-1}^{S}, B=Z_{n-1}$ and the subspace $\Lambda=\Lambda_{n}$ in $H_{1} \cap \ldots \cap H_{n}$ we find a neighbourhood $U_{n-1} \subset U$ of zero in $\mathbb{C}^{m}$, representatives of the germs $\mathbf{d}_{\Lambda_{n}, Z_{n-1}-Z_{n-1}^{S}}, \mathbf{d}_{\Lambda_{(n-1, i)}, Z_{n-1}-Z_{n-1}^{S}}$ and regular directions $\Lambda_{(n-1,1)}, \ldots, \Lambda_{\left(n-1, s_{n}\right)} \in G^{m-k}\left(H_{1} \cap \ldots \cap H_{n-1}\right)$ for $\left|Z_{n-1}\right|$ in $H_{1} \cap \ldots \cap H_{n-1}$ such that if $z \in U_{n-1} \cap H_{1} \cap \ldots \cap H_{n} \subset H_{1} \cap \ldots \cap H_{n-1}$ then

(i) $d_{\Lambda_{n}, Z_{n-1}-Z_{n-1}^{S}}(z) \leq \widetilde{c}_{n-1} \sum_{i=1}^{s_{n}} d_{\Lambda_{(n-1, i)}, Z_{n-1}-Z_{n-1}^{S}}(z)$,

(ii) $\nu\left(\left|Z_{n-1}\right| \cdot \Lambda_{(n-1, i)}, 0\right)=\nu\left(\left|Z_{n-1}\right|, 0\right)$.

(2) Applying Theorem 3.5 for $A=Z_{n-2}-Z_{n-2}^{S}, B=Z_{n-2}$ and $\Lambda=$ $\Lambda_{(n-1, i)}$ in $H_{1} \cap \ldots \cap H_{n-1}$ we find a neighbourhood $U_{(n-2, i)} \subset U_{n-1}$ of zero in $\mathbb{C}^{m}$, representatives of the germs

$$
\mathbf{d}_{\Lambda_{(n-1, i), Z_{n-2}-Z_{n-2}^{S}}} \text { and } \mathbf{d}_{\Lambda_{(n-2, i, j)}, Z_{n-2}-Z_{n-2}^{S}}
$$

and regular directions $\Lambda_{(n-2, i, 1)}, \ldots, \Lambda_{\left(n-2, i, s_{(n-1, i)}\right)} \in G^{m-k}\left(H_{1} \cap \ldots \cap\right.$ $\left.H_{n-2}\right)$ for $\left|Z_{n-2}\right|$ in $H_{1} \cap \ldots \cap H_{n-2}$ such that if $z \in U_{(n-1, i)} \cap H_{1} \cap \ldots \cap$ $H_{n-1} \subset H_{1} \cap \ldots \cap H_{n-2}$ then

(i) $d_{\Lambda_{(n-1, i)}, Z_{n-2}-Z_{n-2}^{S}}(z) \leq \widetilde{c}_{(n-2, i)} \sum_{j=1}^{s_{(n-1, i)}} d_{\Lambda_{(n-2, i, j)}, Z_{n-2}-Z_{n-2}^{S}}(z)$,

(ii) $\nu\left(\left|Z_{n-2}\right| \cdot \Lambda_{(n-2, i, j)}, 0\right)=\nu\left(\left|Z_{n-2}\right|, 0\right)$.

Define $U_{(n-2)}=\bigcap_{i=1}^{s_{n}} U_{(n-2, i)}, \widetilde{c}_{n-2}=\max _{i} \widetilde{c}_{(n-2, i)}, s_{n-1}=\max _{i} s_{(n-1, i)}$.

Inductively at step $(l)$ we make the following choice:

(l) Applying Theorem 3.5 for $A=Z_{n-l}-Z_{n-l}^{S}, B=Z_{n-l}$ and $\Lambda=$ $\Lambda_{(n-l+1, I)}$ in $H_{1} \cap \ldots \cap H_{n-l+1}$ we find a neighbourhood $U_{(n-l, I)} \subset U_{n-l-1}$ of zero in $\mathbb{C}^{m}$, representatives of the germs

$$
\mathbf{d}_{\Lambda_{(n-l-1, I)}, Z_{n-l}-Z_{n-l}^{S}} \text { and } \mathbf{d}_{\Lambda_{(n-l, I, j)}, Z_{n-l}-Z_{n-l}^{S}}
$$

and regular directions $\Lambda_{(n-l, I, 1)}, \ldots, \Lambda_{\left(n-l, I, s_{(n-l+1, i)}\right)} \in G^{m-k}\left(H_{1} \cap \ldots \cap\right.$ $\left.H_{n-l}\right)$ for $\left|Z_{n-l}\right|$ in $H_{1} \cap \ldots \cap H_{n-l}$ such that if $z \in U_{\left(n-1, I_{l-1}\right)} \cap H_{1} \cap \ldots \cap$ $H_{n-l+1} \subset H_{1} \cap \ldots \cap H_{n-l}$ then

(i) $d_{\Lambda_{(n-l+1, I)}, Z_{n-l}-Z_{n-l}^{S}}(z) \leq \widetilde{c}_{(n-l, I)} \sum_{j=1}^{s_{(n-1+1, I)}} d_{\Lambda_{(n-l, I, j)}, Z_{n-l}-Z_{n-l}^{S}}$,

(ii) $\nu\left(\left|Z_{n-l}\right| \cdot \Lambda_{(n-l, I, j)}, 0\right)=\nu\left(\left|Z_{n-l}\right|, 0\right)$.

Define $U_{(n-l)}=\bigcap_{I_{l-1}} U_{(n-l, I)}, \widetilde{c}_{n-l}=\max _{I} \widetilde{c}_{(n-l, I)}$ and $s_{n-l+1}=$ $\max _{I} s_{(n-l+1, I)}$.

Finally in the last $n$th step we get a certain number $s_{1}$ of linear subspaces $\Lambda_{(0, I)}\left(I=I_{n}=\left(i_{1}, \ldots, i_{n}\right)\right)$, which are the regular directions for $\left|Z_{0}\right|$ in 
$\mathbb{C}^{m}$. From Proposition 3.7 applied to the cycle $Z_{0}$ we get, for $I=I_{n}$,

$$
\varrho(z, Z)=\varrho\left(z,\left|Z_{0}\right|\right) \geq \widetilde{c} \mathbf{d}_{\Lambda_{(0, I)}, Z_{0}}(z)
$$

for some constant $\widetilde{c}>0$, independent of $I$, and for $z \in W_{(0, I)}$, where $W_{(0, I)} \subset$ $U_{0}$ is a neighbourhood of zero in $\mathbb{C}^{m}$. Put $W=\bigcap_{I} W_{(0, I)}$.

Fix now $z \in W \cap S$. As $z \in H_{1} \cap \ldots \cap H_{n}$, Remark 3.1 shows that for some representatives of the germs $\mathbf{d}_{\Lambda_{n}, Z_{n-1}-Z_{n-1}^{S}}$ and $\mathbf{d}_{\Lambda_{n}, Z_{n}^{S}}$ we have

(i) $d_{\Lambda_{n}, Z_{n-1}-Z_{n-1}^{S}}(z)=d_{\Lambda_{n}, Z_{n}^{S}}(z)$,

(ii) $\varrho(z, Z \cap S)^{\alpha_{n}} \leq d_{\Lambda_{n}, Z_{n}^{S}}(z)$ for $\alpha_{n}=\nu\left(Z_{n}^{S}, 0\right)$.

We will choose a special subspace from each of the systems $\Lambda_{\left(l, I_{n-l}\right)}$.

(1) For the point $z$ choose from the system $\Lambda_{(n-1,1)}, \ldots, \Lambda_{\left(n-1, s_{n}\right)}$ the maximal subspace $\Lambda_{n-1}(z)=\Lambda_{\left(n-1, i_{0}\right)}$ (Remark 3.6). We get

$$
d_{\Lambda_{n}, Z_{n-1}-Z_{n-1}^{S}}(z) \leq c_{n-1} d_{\Lambda_{n-1}(z), Z_{n-1}-Z_{n-1}^{S}}(z)
$$

where $c_{n-1}=\widetilde{c}_{n-1} s_{n}$.

Since $z \in H_{1} \cap \ldots \cap H_{n-1}$, for some representatives of the germs $\mathbf{d}_{\Lambda_{n-1}, Z_{n-1}-Z_{n-1}^{S}}, \mathbf{d}_{\Lambda_{n-1}, Z_{n-1}^{S}}$ and $\mathbf{d}_{\Lambda_{n-1}, Z_{n-2}-Z_{n-2}^{S}}$ we have

(i) $d_{\Lambda_{n-1}(z), Z_{n-1}-Z_{n-1}^{S}}(z) \cdot d_{\Lambda_{n-1}(z), Z_{n-1}^{S}}(z)=d_{\Lambda_{n-1}(z), Z_{n-2}-Z_{n-2}^{S}}(z)$,

(ii) $\varrho(z, Z \cap S)^{\alpha_{n-1}} \leq d_{\Lambda_{n-1}(z), Z_{n-1}^{S}}(z)$ for $\alpha_{n-1}=\nu\left(Z_{n-1}^{S}, 0\right)$.

Combining the properties $(* *)$, (i), (ii) we have

$$
\varrho(z, Z \cap S)^{\alpha_{n}+\alpha_{n-1}} \leq c_{n-1} d_{\Lambda_{n-1}(z), Z_{n-2}-Z_{n-2}^{S}}(z) .
$$

(l) Having $\Lambda_{n-1}(z), \ldots, \Lambda_{n-l+1}(z)=\Lambda_{\left(n-l+1, I_{l-1}\right)}$ we choose from the system $\Lambda_{\left(n-l, I_{l-1}, 1\right)}, \ldots \Lambda_{\left(n-l, I_{l-1}, s_{I_{l}-1}\right)}$ the maximal subspace $\Lambda_{n-l+1}(z)$ and we get

$$
d_{\Lambda_{n-l+1}(z), Z_{n-l}-Z_{n-l}^{S}}(z) \leq c_{n-l} d_{\Lambda_{n-l}(z), Z_{n-l}-Z_{n-l}^{S}}(z),
$$

where $c_{n-l}=\widetilde{c}_{n-l} s_{n-l+1}$.

As $z \in H_{1} \cap \ldots \cap H_{n-l}$ we can repeat the same observation:

(i) $d_{\Lambda_{n-l}(z), Z_{n-l}-Z_{n-l}^{S}}(z) \cdot d_{\Lambda_{n-l}, Z_{n-l}^{S}}(z)=d_{\Lambda_{n-l}(z), Z_{n-l-1}-Z_{n-l-1}^{S}}(z)$,

(ii) $\varrho(z, Z \cap S)^{\alpha_{n-l}} \leq d_{\Lambda_{n-l}(z), Z_{n-l}^{S}}(z)$ for $\alpha_{n-l}=\nu\left(Z_{n-l}^{S}, 0\right)$.

Combining $(* *)$, (i), (ii) we obtain

$$
\varrho(z, Z \cap S)^{\alpha_{n}+\ldots+\alpha_{n-l}} \leq c_{n-1} \ldots c_{n-l} d_{\Lambda_{n-l}(z), Z_{n-l-1}-Z_{n-l-1}^{S}}(z) .
$$

After the last step we get

$$
\varrho(z, Z \cap S)^{\alpha_{n}+\ldots+\alpha_{0}} \leq c_{n-1} \ldots c_{0} d_{\Lambda_{0}(z), Z_{0}} .
$$

Since $\alpha_{n}+\ldots+\alpha_{0}=p_{\mathcal{H}}$, applying $(*)$ we finally get $\varrho(z, Z \cap S)^{p_{\mathcal{H}}} \leq c \varrho(z, Z)$ for $c=c_{n-1} \ldots c_{0} \cdot \widetilde{c}^{-1}$. 
The constant $c$ is independent of $z$ so the above inequality holds for all $z \in W \cap S$ and Lemma 4.2 ends the proof.

Corollary 4.5. Let $X$ and $Y$ be analytic subsets of $M$, and $a \in X \cap Y$. Then $X$ and $Y$ are $p$-separated at a with $p=\nu(X \bullet Y, a)$.

Proof. First note that for irreducible analytic subsets $X, Y$ of $M$ we get $\nu(X \bullet Y, a)=g\left(X \times Y, \Delta_{M},(a, a)\right)$. As

$$
p\left(X \times Y, \Delta_{M},(a, a)\right) \leq g\left(X \times Y, \Delta_{M},(a, a)\right),
$$

Theorem 4.4 and Lemma 4.3 imply that $X$ and $Y$ are $\nu(X \bullet Y, a)$-separated at $a$.

Now consider arbitrary $X, Y$ analytic in $M$ and let $W$ be an open neighbourhood of $a$ such that

$$
X \cap W=X_{1} \cap W \cup \ldots \cup X_{k} \cap W, \quad Y \cap W=Y_{1} \cap W \cup \ldots \cup Y_{l} \cap W
$$

where $X_{i}, Y_{j}$ are irreducible components of $X, Y$ respectively such that $a \in$ $X_{i} \cap Y_{j}$ for all $i, j$. Then $X_{i}$ and $Y_{j}$ are $p_{i j}=\nu\left(X_{i} \bullet Y_{j}, a\right)$-separated at $a$. Take a chart $(\varphi, \Omega)$ of $M$ such that $\Omega \subset W, \varphi(\Omega) \subset B(0,1) \subset \mathbb{C}^{m}$ and $\varphi(a)=0$. Then, as $\varphi\left(\Omega \cap X_{i}\right)$ and $\varphi\left(\Omega \cap Y_{j}\right)$ are $p_{i j}$-separated at zero, there exist $U_{i j} \subset \Omega$ and $c_{i j}>0$ such that for $x \in X_{i} \cap U_{i j}$ we have

$$
\varrho\left(\varphi(x), \varphi\left(Y_{j} \cap \Omega\right)\right) \geq c_{i j} \varrho\left(\varphi(x), \varphi\left(X_{i} \cap Y_{j} \cap \Omega\right)\right)^{p_{i j}} .
$$

Take $U=\bigcap U_{i j}, c=\min _{i j} c_{i j}$ and fix $x \in U \cap X$. There exist $i, j$ such that $x \in U \cap X_{i}$ and $\varrho(\varphi(x), \varphi(Y \cap \Omega))=\varrho\left(\varphi(x), \varphi\left(Y_{j} \cap \Omega\right)\right)$. We get

$$
\begin{aligned}
\varrho(\varphi(x), \varphi(Y \cap \Omega)) & =\varrho\left(\varphi(x), \varphi\left(Y_{j} \cap \Omega\right)\right) \geq c \varrho\left(\varphi(x), \varphi\left(X_{i} \cap Y_{j} \cap \Omega\right)\right)^{p_{i j}} \\
& \geq c \varrho(\varphi(x), \varphi(X \cap Y \cap \Omega))^{p_{i j}} \\
& \geq c \varrho(\varphi(x), \varphi(X \cap Y \cap \Omega))^{\sum_{i, j} p_{i j}} .
\end{aligned}
$$

As $\sum_{i j} p_{i j}=\nu(X \bullet Y, a)$ Lemma 4.2 ends the proof.

Let us recall that for isolated intersection one can separate the sets $X$ and $Y$ with a better exponent, $p=\nu(X \cdot Y, a)-\nu(X, a) \cdot \nu(Y, a)+1$ (see $\left.\left[\mathrm{T}_{3}\right]\right)$. The following example shows that even in the situation of proper but not isolated intersection we cannot improve Corollary 4.5.

EXAMPLE 4.6. In $\mathbb{C}^{3}$ take the intersection of $X=\left\{(x, y, z) \in \mathbb{C}^{3}\right.$ : $\left.x^{2}+x y+z^{2}=0\right\}$ with the subspace $Y=\left\{(x, y, z) \in \mathbb{C}^{3}: x=0\right\}$.

It is easy to verify that $\nu(X \cdot Y, 0)=\nu(X, 0)=2, \nu(Y, 0)=1$ and the best separation exponent for these two sets at zero is 2 .

Acknowledgements. I am extremely grateful to Piotr Tworzewski for his constant help and several valuable remarks. I also wish to thank the University of Lille, where the great part of this paper was prepared, for the invitation and hospitality. 


\section{References}

[ATW] R. Achilles, P. Tworzewski and T. Winiarski, On improper isolated intersection in complex analytic geometry, Ann. Polon. Math. 51 (1990), 21-36.

[Ch] E. M. Chirka, Complex Analytic Sets, Kluwer Acad. Publ., 1989.

[CKT] E. Cygan, T. Krasiński and P. Tworzewski, Separation at infinity and Eojasiewicz exponent of polynomial mappings, preprint IMUJ 1997/22, 1-9.

[CT] E. Cygan and P. Tworzewski, Proper intersection multiplicity and regular separation of analytic sets, Ann. Polon. Math. 59 (1994), 293-298.

[D] R. Draper, Intersection theory in analytic geometry, Math. Ann. 180 (1969), 175-204.

[JKS] S. Ji, J. Kollár and B. Shiffman, A global Eojasiewicz inequality for algebraic varieties, Trans. Amer. Math. Soc. 329 (1992), 813-818.

[K] J. Kollár, Sharp effective Nullstellensatz, J. Amer. Math. Soc. 1 (1988), 963975.

$\left[\mathrm{E}_{1}\right] \quad$ S. Łojasiewicz, Ensembles semi-analytiques, I.H.E.S., Bures-sur-Yvette, 1965.

$\left[\mathrm{E}_{2}\right]$ - Introduction to Complex Analytic Geometry, Birkhäuser, Basel, 1991.

$\left[\mathrm{E}_{3}\right]$ - - Sur la séparation régulière, Univ. Studi Bologna Sem. Geom. 1985, 119-121.

$\left[\mathrm{T}_{1}\right] \quad \mathrm{P} . \mathrm{Tw}$ or zewski, Intersection of analytic sets with linear subspaces, Ann. Scuola Norm. Sup. Pisa (4) 17 (1990), 227-271.

$\left[\mathrm{T}_{2}\right] \quad$ - Intersection theory in complex analytic geometry, Ann. Polon. Math. 62 (1995), 177-191.

$\left[\mathrm{T}_{3}\right] \quad-$, Isolated intersection multiplicity and regular separation of analytic sets, Ann. Polon. Math. 58 (1993), 213-219.

$\left[\mathrm{TW}_{1}\right]$ P. Tworzewski and T. Winiarski, Analytic sets with proper projections, J. Reine Angew. Math. 337 (1982), 68-76.

$\left[\mathrm{TW}_{2}\right]-,-$, Cycles of zeroes of holomorphic mappings, Bull. Polish Acad. Sci. Math. 37 (1989), 95-101.

[W] T. Winiarski, Continuity of total number of intersection, Ann. Polon. Math. 47 (1986), 155-178.

Institute of Mathematics

Jagiellonian University

Reymonta 4

30-059 Kraków, Poland

E-mail: cygan@im.uj.edu.pl 